\title{
La Ortopedia infantil para pediatras de Atención Primaria
}

\author{
AD. Delgado Martínez $z^{a}, M D$. del Castillo Codes ${ }^{b}$ \\ aDirector de la Unidad de Gestión Clínica de Traumatología. Hospital San Agustín. \\ Linares, Jaén. Coordinador del área de Cirugía. Universidad de Jaén. Jaén. España. \\ 'Pediatra. CS San Felipe. Jaén. España.
}

La formación en patología del aparato locomotor en Pediatría es deficitaria. Es cierto que la Pediatría es una de las especialidades más extensas dentro de la medicina, solo hay que echar un vistazo al tamaño de los libros más habituales de la especialidad para entenderlo'. Sin embargo, en estos textos se ha tratado tradicionalmente de forma muy superficial toda la patología referente al aparato locomotor ${ }^{1}$ $y$, lo que es aún peor, lo poco que se trata se hace desde la perspectiva del "superespecialista", desarrollando temas de muy poca frecuencia o con una visión excesivamente quirúrgica. Además, durante el periodo de formación MIR en Pediatría, se dedica muy poco tiempo a la formación en Ortopedia (por no decir ninguno). Tradicionalmente, esta especialidad se ha considerado muy quirúrgica y, por ello, se le ha dado poco protagonismo durante la formación MIR. Sin embargo, el diagnóstico de los problemas ortopédicos tan frecuentes en el niño deben hacerlo los pediatras de Atención Primaria (AP). Esto supone un "choque" cuando el pediatra comienza su actividad en el centro de salud, pues se encuentra ante muchas enfermedades que apenas ha visto durante la residencia y que debe diagnosticar y muchas veces tratar.

Por ello, la Ortopedia y Traumatología infantil es una de las parcelas que más preocupan al pediatra de AP. En primer lugar, es uno de los motivos de consulta más frecuentes en las consultas infantiles de los centros de salud. En segundo lugar, sigue existiendo (por los motivos previamente indicados) mucho desconocimiento tanto en el diagnóstico como en el tratamiento de estas entidades. Hay cuadros (como la displasia del desa-

Alberto D. Delgado Martínez, adelgado@ujaen.es

Los autores declara no presentar conflictos de intereses en relación con la preparación y publicación de este artículo. 
rrollo de la cadera) que pueden evolucionar bien o mal dependiendo de su diagnóstico precoz, mientras que otros, como el síndrome de hiperpresión rotuliana externa, pueden doler de forma indefinida si no se les aplica el tratamiento adecuado (tan simple como algunos ejercicios y medidas posturales). En tercer lugar, tampoco es posible derivar a todos los pacientes al traumatólogo. Por todo ello, el pediatra de AP sufre a diario una gran ansiedad en su práctica diaria. Toda esta preocupación se pone de manifiesto cuando se observa que los talleres y cursos específicos de Ortopedia infantil para pediatras de AP son de los primeros en completarse todos los años en el curso anual de la Asociación Española de Pediatría de Atención Primaria (AEPap).

Pero, ¿cómo formarse en Ortopedia infantil? Obviamente, lo primero sería que se permitiera (al menos) la rotación por las consultas externas de los Servicios de Ortopedia Infantil a los MIR de Pediatría, quienes probablemente no necesiten una rotación muy extensa (incluso 15 días podrían ser suficientes) para familiarizarse de forma básica con estas patologías. Para el especialista ya formado, existen millones de páginas en Internet y libros extensísimos con todas las patologías en este campo, pero tanta información hace que se pierda lo básico, lo esencial. No tenemos tiempo para leer cientos de páginas que nos proporcionan escasa información útil. Por ello, es importante apoyar e impulsar el desarrollo de actividades (cursos, talleres, artículos, libros, etc.) muy básicas, resumidas, fáciles y rápidas de comprender y asimilar en la práctica clínica diaria, diseñadas específicamente para el pediatra de AP. En este punto, la AEPap juega un papel fundamental, promocionando todo este tipo de actividades.

Respecto a los problemas actuales de interés para los pediatras, aún se sigue debatiendo la necesidad o no de un cribado ecográfico generalizado a todos los neonatos para prevenir la displasia del desarrollo de la cadera². Sin embargo, estudios recientes ${ }^{3}$ han vuelto a confirmar que la mejor manera de conseguir una cadera no artrósica e indolora hasta los 60 años de edad es la realización de una exploración clínica seriada a todos los niños (lo que suele hacerse en el Programa de Salud Infantil), y realizar la ecografía solo a pacientes seleccionados (con factores de riesgo o exploración anormal o dudosa). En Estados Unidos, la exploración clínica sistemática de la cadera en los primeros meses de edad sigue siendo la técnica principal de cribado ${ }^{4}$; la ecografía debería realizarse a las cuatro o a 
las seis semanas y no antes, porque la prueba tiene poca especificidad antes de dicho periodo 5 . Aunque sigue siendo un tema no completamente resuelto, el trabajo de los doctores Lovera et al. ${ }^{6}$, publicado en este número, ahonda en esta discusión.

En este número encontramos otros cuatro excelentes artículos sobre Ortopedia infantil: los doctores Álvarez y Núñez presentan una excelente revisión sobre la escoliosis, indicando que la escoliosis no duele (si es así hay que buscar otros diagnósticos) y la importancia del test de Adams como criterio de derivación: es necesario reevaluar en seis meses si el test está entre 5 y $9^{\circ}$, y remitir al especialista si es mayor de $10^{\circ}$. Los doctores Parra y Bueno ${ }^{8}$, en su artículo sobre el pie plano, destacan la alta frecuencia del pie plano valgo flexible en la infancia, y el problema de las plantillas, que actualmente no se recomiendan en esta patología, pero muchas veces la familia u otro profesional insiste en ellas. En este contexto, la coalición tarsiana es uno de los primeros cuadros a diferenciar ante un pie plano que no corrige de puntillas, con una maniobra tan simple como la movilidad pasiva subastragalina ${ }^{9}$, que siempre está (en mayor o menor grado, según la edad) limitada en estos niños. Los doctores Carabaño y Llorente ${ }^{10}$, a través de la presentación de un caso clínico de la tan frecuente y conocida enfermedad de OsgoodSchlatter, ofrecen una excelente, clara y concisa explicación del tratamiento de dicha enfermedad, basado en estiramientos y modificaciones de la actividad, un tratamiento tan simple como efectivo para evitar las tan temidas recaídas o el abandono precoz de la práctica deportiva de un niño para el que el deporte es fundamental. Por último, la doctora Ortega y colaboradores ${ }^{11}$ presentan otro caso de dedo en gatillo infantil, cuadro que aparece sobre todo en el primer dedo, en los dos primeros años de edad. Aunque hay algunos casos que se resuelven con tratamiento médico, casi todos precisan la liberación quirúrgica de la polea, que es una intervención rápida, sencilla y casi sin complicaciones, que resuelve el cuadro de forma definitiva ${ }^{12}$. 


\section{Bibliografía}

1. Kliegman RM, Behrman RE, Jenson $H B$, Stanton JH. Nelson Tratado de Pediatría, 18. ${ }^{a}$ ed. Elsevier; 2008.

2. Kim YJ, Noonan KJ. What's new in pediatric orthopaedics. J Bone Joint Surg Am. 2010;92: 1575-82.

3. Mahan ST, Katz JN, Kim YJ. To screen or not to screen? A decision analysis of the utility of screening for developmental dysplasia of the hip. J Bone Joint Surg Am. 2009;91:1705-19.

4. Matheney TH, Kim YJ. Hip, Pelvis, and Femur disorders: Pediatrics. En: Fishgrund JS (ed.). Orthopaedic Knowledge Update 9. Rosemont: Ed. AAOS; 2008. p. 715-26.

5. Choi PD. Pediatric Hip conditions. En: Lieberman JR. AAOS comprehensive orthopaedic review. Rosemont: Ed. AAOS; 2009. p. 321-8.

6. Paz Lovera $M$, Llorente Otones $L$, Rivero Calle I, Lesmes Moltó L, Rodríguez Díaz MR, Rivero Martín MJ. Displasia evolutiva de cadera: controversia sobre el cribado ecográfico universal. Rev Pediatr Aten Primaria. 2011;13: 127-34.

7. Álvarez García de Quesada LI, Núñez Giralda A. Escoliosis idiopática. Rev Pediatr Aten Primaria. 2011;13:135-46.

8. Parra García JI, Bueno Sánchez A. El pie plano; las recomendaciones del traumatólogo infantil al pediatra. Rev Pediatr Aten Primaria. 2011; 13:113-25.

9. Delgado Martínez AD, Marchal Corrales JA. Manual de anatomía funcional y exploración clínica del aparato locomotor, 2. ${ }^{a}$ ed. Jaén: Ed. CaeAla; 2010.

10. Carabaño Aguado I, Llorente Otones L. Enfermedad de Osgood-Schlatter: deporte, adolescencia y dolor. Rev Pediatr Aten Primaria. 2011;13:93-7.

11. Ortega Pérez $S$, Aguilar Sánchez $M$, Cañizo Fernández D, Guerra García de Celis A, Pérez Pérez A. Dedo en resorte congénito en un lactante. Rev Pediatr Aten Primaria. 2011;13:175-7.

12. Herring JA. Tachdjian's Pediatric Orthopaedics, 3.rd ed. Philadelphia: Ed. Saunders; 2002. 